\title{
A Rose by Any Other Name? The Potential Consequences of Microglial Heterogeneity During CNS Health and Disease
}

\author{
Monica J. Carson, Tina V. Bilousova, Shweta S. Puntambekar, Benoit Melchior, \\ Jonathan M. Doose, and Iryna M. Ethell \\ Division of Biomedical Sciences, Center for Glial-Neuronal Interactions, University of California Riverside, Riverside, California
}

Summary: Microglial activation and macrophage infiltration into the CNS are common features of CNS autoimmune disease and of chronic neurodegenerative diseases. Because these cells largely express an overlapping set of common macrophage markers, it has been difficult to separate their respective contributions to disease onset and progression. This problem is further confounded by the many types of macrophages that have been termed microglia. Several approaches, ranging from molecular profiling of isolated cells to the generation of irradiation chimeric rodent models, are now beginning to generate rudimentary definitions distinguishing the various types of microglia and macrophages found within the CNS and the potential roles that these cells may play in health and disease. Key Words: neuroinflammation, TREM, microglia, Alzheimer's disease, multiple sclerosis, purinergic receptors, P2Y12.

\section{INTRODUCTION}

The CNS is primarily composed of neurons and macroglia (oligodendrocytes and astrocytes), all of neuroectodermal origin. ${ }^{1,2}$ Additionally, although the CNS is an immune-privileged site, $\sim 15 \%$ of the cells in the normal healthy CNS are of mesenchymal origin and express most common macrophage markers. ${ }^{3}$ Histologically, these unactivated macrophages are readily detected in all parenchymal and perivascular regions of the healthy brain and spinal cord, as well as in the choroid plexus, dura mater, and leptomeninges. ${ }^{3,4}$ In many studies, all the CNS macrophage populations that display a stellate morphology are classified together as a single resident tissue macrophage population referred to as microglia. Using similar morphological criteria, macrophages with amoeboid morphologies are alternatively designated either as inflammatory macrophages recently derived from the blood or as fully activated microglia., 3,4

This simple morphological classification based on stellate morphology and presence within the CNS has led to some confusion in the literature as to the intent and consequence of labeling cells as microglia. Namely, should all stellate cells within the CNS be classified as

Address correspondence and reprint requests to: Dr. M.J. Carson and Dr. I.M. Ethell, Division of Biomedical Sciences, Center for GlialNeuronal Interactions, University of California-Riverside, Riverside, CA 92521. E-mail: monica.carson@ucr.edu; iryna.ethell@ucr.edu. microglia without respect to their recent history or source (e.g., from blood or from sites within the CNS)? Does this universal label imply that all cells, regardless of their source, have nearly identical functions upon entering the CNS microenvironment? In this view, do morphological parameters merely reflect environmentally modulated changes in any myeloid cell found within the CNS? Alternatively, should the terms 'microglia' and 'macrophage' be used, respectively, to distinguish cells that have resided long-term within the CNS from those that have recently infiltrated?

Although these distinctions are in part semantic, the underlying issue of recent history and source of CNSresident macrophage populations is not merely one of academic distinctions. Rather, this issue has two implications for how CNS-resident macrophages may contribute toward CNS function and dysfunction. First, whether the source of stellate or amoeboid macrophages (i.e., the microglia) is from the blood reflects their ability to be targeted by pathogens and drugs (both therapeutic and those of abuse) in the blood before they enter the CNS. ${ }^{3,4}$ Second, whether the source is largely from a self-renewing or long-lived source located within the CNS reflects the time span within which these cells have adapted to homeostatic signals from the CNS environment.

In sections that follow, we discuss recent studies defining the types of microglia and macrophages found within the CNS, their differential sources, and the role of 
the CNS in defining the phenotype and functions of these cells. Understanding the source of the heterogeneity of CNS-resident macrophages is likely to provide new opportunities for modulating CNS pathogenesis.

\section{MICROGLIAL ACTIVATION}

An early biowarning or primary cause of disease?

Before the identities of CNS-resident macrophages and microglia are defined, it is important first of all to ask why we should focus on these cells in order to understand CNS health and disease? For nearly a century, microglial activation and macrophage infiltration, singly or in combination, have been widely recognized as correlating with the earliest signs of detectable pathology and/or dysfunction in the CNS. ${ }^{5-9}$ In response to changes in neuronal activity, pathogen encounter, or mechanical injury, microglia display dramatic changes in cell morphology and increased expression of standard macrophage activation markers. ${ }^{5-10}$

More recently, microglial activation has become a recognized precursor of pathology in otherwise normalappearing regions of the CNS. A notable example of this phenomenon is observed in multiple sclerosis, an autoimmune disorder characterized by demyelinated lesions. ${ }^{11-13}$ Prominent microglial activation is easily detected in areas of active demyelination or active lymphocyte infiltration. However, by histopathology and by in vivo live imaging methods, microglial activation can be observed in cortical gray matter regions and in normal-appearing white matter regions that are distant from the areas of active demyelination. ${ }^{13-15}$ Such observations raise the question of whether microglial activation is a noncausative biomarker of otherwise covert, early neuronal damage or whether microglial activation precedes and is a causal factor for subsequent CNS histopathology.

In vivo and in vitro data demonstrate the potential of microglia to play both beneficial and detrimental roles. ${ }^{16,17}$ Whether in vitro or in vivo, blocking neuronal activity with tetrodotoxin immediately leads to increased microglial expression of major histocompatibility complex (MHC) class II. ${ }^{10,18,19}$ Healthy neurons express several molecules, such as CD200 and CD22, that actively downregulate microglial and macrophage activation state. $^{20-22}$ The consequence of decreased expression of these molecules in injured or diseased neurons has been demonstrated in knockout mice lacking these molecules. For example, in CD200 or CD200 receptor-deficient mice, the basal activation states of all macrophage populations (including those in the CNS) are increased, as judged by altered morphology and increased expression of MHC and CD11b. ${ }^{21,22}$ Furthermore, inflammatory reactions following mechanical injury, exposure to toll-like receptor ligands such as lipopolysaccharide (LPS) as well as the progression of experimental autoimmune encephalomyelitis (EAE) are dramatically exacerbated in the absence of these inhibitory cues. ${ }^{21,22}$ Targeted development of inhibitory therapies that mimic these examples of active neuronal inhibition will rely on defining two key parameters. What is the basal phenotype and function of unactivated microglia in the healthy CNS? And is this phenotype essentially identical throughout the CNS?

\section{THE BIRD'S EYE VIEW OF MICROGLIA IN THE HEALTHY CNS}

Until very recently, the basal phenotype of resident microglia in the healthy brain was considered to be uniformly quiescent and inactive. ${ }^{18,23,24}$ Two recent studies published by Nimmerjahn et al. ${ }^{18}$ and by Davalos et al. ${ }^{24}$ have dramatically altered this viewpoint. In both studies, all CNS macrophage populations were visualized in vivo by transgenic expression of fluorescent molecules. Using two-photon imaging through either a thinned portion or a cut window in the skull, both groups found that, although microglial cell bodies remained relatively fixed, their thin ramified processes were remarkably motile. Process formation and withdrawal was continually ongoing in the absence of any pathogenic stimulus and that the average basal motility of microglial processes was higher than that of astrocytes. From the rate of observed process remodeling, it was apparent that each CNS-macrophage could survey all elements of the CNS every 6 hours. ${ }^{18,23,24}$ Nimmerjahn et al. ${ }^{18}$ also noted that a subset of microglial processes were less motile and provided a stable scaffold, perhaps anchoring the microglia in place. These data suggest that the formation of microglial processes in the healthy CNS may not be random and may serve to integrate homeostatic signals throughout the entire CNS.

Although such studies convincingly demonstrate robust microglial activity in the normal CNS, they do not fundamentally alter the debate about the function of CNS-resident macrophages. Namely, do these cells only play a surveillance function in the healthy CNS? (This would mean that these cells, though quite important, are essentially inactive in the absence of pathogens and injury.) Or do these cells play more active roles in maintaining optimal neuronal function?

In part, these debates have been difficult to resolve because many studies, including the two just described, experimentally group all CNS-resident macrophages together regardless of their source or location in the CNS. Thus, if CNS-resident macrophages are heterogeneous in source and function, such an approach may confound or obscure regional or cell-type specific functions. 


\section{A ROSE BY ANY OTHER NAME. .?}

Because the CNS is an immune-privileged site, for most of the last century it was debated whether it is even possible for peripheral macrophage populations to enter the healthy CNS and contribute to the CNS-resident macrophage population. ${ }^{4,25,26}$ Consequently, many researchers tested whether the macrophages found within the healthy CNS were of neuroectodermal or mesenchymal origin. Over the past 25 years, numerous approaches have demonstrated that the stem cells or progenitors that give rise to neurons and macroglia do not give rise to microglia, neither in vitro or in vivo. ${ }^{27}$

Although CNS-resident macrophage populations can transiently express macroglial markers such as NG2 or GD3 during neuroinflammation and regeneration, a neuroectodermal source for any subpopulation these cells has never been demonstrated. ${ }^{27}$ Rather, the CNS appears to be seeded by cells of hematopoietic (mesenchymal) origin early in CNS development. In rodents, microglia can be detected by at least as early as embryonic day 10.5. ${ }^{26,28,29}$ However, histological data also suggest that more than one population may seed the CNS. ${ }^{26,29} \mathrm{Neo}-$ natally, microglia are not as abundant as in the adult CNS. Indeed, fountainheads of microglia are evident infiltrating the hippocampus and other CNS structures shortly after birth in the rodent CNS. ${ }^{1,30}$

Several groups have also tested whether influxes of macrophages from peripheral sources into the CNS continue through adulthood. To this end, some groups have directly tested whether in vitro differentiated microglia can home to the CNS following intravenous injection. ${ }^{31}$ The injected cells were indeed found to preferentially home to the CNS (primarily the hippocampus and corpus callosum); however, these studies did not determine how long these cells remained in the CNS, nor whether they fully integrated into the CNS parenchyma. ${ }^{31}$ These studies demonstrate the potential of a small number of differentiated microglia to traffic across an intact bloodbrain barrier into the CNS, but do not address whether the bone marrow routinely generates cells able to preferentially home to the CNS and then differentiate into microglia.

Several other groups, using irradiation bone marrow chimeric approaches, have demonstrated that the CNSresident macrophage population is likely to have two global sources and to have two very different lifespans or trafficking kinetics. ${ }^{32-38}$ In this methodology, rodents are treated with sufficient doses of irradiation to destroy their bone marrow, but not so high a dose as to cause subsequent systemic inflammation. Irradiated rodents are supplemented with donor bone marrow with a genotype differing from the host. Most often, the donor bone marrow expresses an easily detectable transgene such as green fluorescent protein (GFP). Estimates of cell life- span are determined by monitoring the rates that macrophages with the host genotype disappear and the rate that macrophages with the donor genotype appear (e.g., the rate that GFP-positive cells appear).

With such a methodology, one population of CNS macrophages is found to be replaced from bone marrow sources frequently (approximately every 2 weeks), and a second population is found to be rarely replaced from the bone marrow and thus is likely largely self-renewing or long-lived (or both). ${ }^{28,32-38}$ The former, short-lived population consists of CNS-resident macrophages located in perivascular regions, the cerebellum, rostral stream, and olfactory bulb. In the literature, these cells are often referred to as either perivascular macrophages or microglia. ${ }^{28,32-38}$ Parenchymal macrophage populations in all other regions of the CNS belong largely to the latter population and in the literature are most often referred to simply as microglia. ${ }^{28,32-38}$

Irradiation is not a neutral treatment and results in long-term alterations in the vasculature even when the heads are shielded from irradiation, likely a consequence of the cytokine storm associated with death of bone marrow and tissue macrophages throughout the body. ${ }^{28,32-38}$ It has been suggested in studies of peripheral macrophages that irradiation chimeric methodologies cause premature death and turnover and thus result in overestimates of tissue macrophage turnover. Furthermore, several studies using these methods have found that the replacement macrophage populations within the CNS do not express the same phenotype and level of activation markers observed in unmanipulated mice. Instead, most often the replacement cells are reported to express markers such as the dendritic cell marker, CD11c which are not expressed by resident macrophages populations in the healthy unmanipulated CNS. ${ }^{28,32-38}$ Although changes in the phenotype of otherwise unstimulated microglia may result from using excessive doses of radiation exposure, thus causing nonspecific infiltration of inflammatory macrophages into the CNS, it may also be an inherent experimental consequence of this type of irradiation methodology.

Despite these limitations, the irradiation chimeric approach has provided a powerful technique for probing the differential contributions of long-term residents of the CNS from those of cells recently entering the CNS. Stalder et al. ${ }^{39}$ used this approach to demonstrate that, in transgenic mouse models of Alzheimer's disease, donorderived macrophages infiltrate the CNS and contribute to the cells surrounding the amyloid plaques. Rivest and colleagues ${ }^{40}$ demonstrated, surprisingly, that donor-derived macrophages do not simply accumulate with age and increasing plaque pathology. Rather, they found that the numbers of donor-derived cells peaked early in disease just at the onset of amyloid plaque deposition (at $\sim 6-9$ months of age). 
Strikingly, the Rivest group found that the donorderived macrophages disappeared from the CNS with time and that their disappearance correlated with increased plaque deposition. ${ }^{40}$ Based on these observations and on in vitro assays of phagocytosis, they concluded that the hematogenously derived (donor-derived) macrophage populations were much more effective phagocytes than the host-derived microglia. They further speculated that plaque deposition was in part due to the failure of the peripheral macrophages to remain within the CNS. Because both the host-derived and donor-derived cells displayed a stellate (though activated) morphology in vivo, these authors referred to both populations as microglia. ${ }^{40}$ Based on their ability to leave the CNS, however, and their failure to establish permanent residency in the CNS, these types of donor-derived cells could have been termed CNS-infiltrating macrophages, to distinguish them from classic host-derived microglia. ${ }^{39}$

A similar division of labor between host-derived microglia and bone marrow-derived macrophages has been observed in the facial axotomy model. In this model, facial motoneuron cell death within the brainstem is modest and similar in wild-type and T-cell-deficient mice during the first 2 weeks following facial axotomy. ${ }^{41,42}$ By 4 weeks after axotomy, however, facial motoneuron cell death is much higher in mice lacking $\mathrm{CD} 4^{+} \mathrm{T}$ cells. Thus, in this model the $\mathrm{CD} 4^{+} \mathrm{T}$ cells play a neuroprotective role in slowing the rate of neuronal death, perhaps allowing time for axonal regrowth and for the new axons to find their target and receive targetderived growth factors.

Byram et al. $^{42}$ used the same irradiation chimeric methodologies used to differentially localize MHC class II expression in the donor and host populations. They found that donor-derived macrophage or dendritic cell populations (or both) were essential to initiating the axotomy-induced T-cell response; however, even though donor-derived macrophages infiltrated the facial motoneuron nucleus within the brainstem, these cells were insufficient to drive the neuroprotective T-cell response. Instead, host-derived microglia were essential to evoke or sustain the neuroprotective response from the $\mathrm{T}$ cells primed by the donor-derived cells. ${ }^{42}$

Taken together, these several studies show that both sources of CNS macrophages can develop cell typespecific effector functions and that both sources of cells are required to maintain optimal CNS function. ${ }^{39,40,42}$

\section{NATURE VERSUS NURTURE}

\section{Are microglia defined by a single uniform phenotype or a specific lineage marker?}

Is this phenotype environmentally determined?

In 1991, Sedgwick and colleagues ${ }^{43}$ reported that CNS-resident microglia expressed leukocyte common antigen (also known as CD45), the same as all other macrophage populations in the body. Unlike macrophages that acutely infiltrate the CNS, however, microglia expressed uniformly lower levels of this molecule. ${ }^{43}$ Irradiation chimeric methodologies confirmed that the relative levels of CD45 could be used to distinguish CNS-resident microglia from acutely infiltrating cells when analyzed by flow cytometry (but not by immunohistochemistry, which lacks sufficient dynamic range of detection).

These seminal observations suggested that stable, global differences in the phenotype of microglia versus acutely infiltrating macrophages did exist. Note, however, that the CD45 levels of small but significant percentages of each population do overlap. It is unclear what drives this differential expression of CD45 or how stable these differences are; upon culture in the absence of neurons, microglial levels of CD45 do rise dramatically. ${ }^{44}$ Furthermore, some reports indicate that the levels of CD45 of peripherally derived CNS-infiltrating macrophages decrease with increasing time within the CNS (although it is unclear whether the levels fully decline to the levels of unactivated resident microglia). ${ }^{45,46}$

More recently, Vallières and colleagues ${ }^{47}$ have reported that $\mathrm{CD} 45^{\text {low }}$ microglia isolated from the healthy CNS express the purinergic receptor P2Y12 at much higher levels than other macrophage populations in spleen or other organs, as analyzed by real-time PCR and in situ hybridization analysis. As part of imaging studies examining microglial responses within living tissues, Haynes et al $^{48}$ found that all macrophage populations within the CNS express the P2Y12 receptor. Because perivascular microglia are being continually replaced from bone marrow-derived sources, the apparent constitutive expression of this molecule again supports CNSdriven acquisition of a CNS-resident phenotype. Whether acutely infiltrating inflammatory macrophages would acquire this phenotype as a function of time remains to be studied.

These studies compellingly illustrate broad differences between resident and acutely infiltrating macrophage populations in the CNS and may, at first glance, suggest that CNS-resident macrophages are a largely homogeneous population. However, a simple histological analysis of microglial morphology and density as a function of brain region suggests there is not a single global microglial phenotype. For example, using F4/80 monoclonal antibodies to label all CNS macrophage populations, Lawson et al. ${ }^{49,50}$ observed that the regional density and shape of microglia varied as a function of brain region. Microglia with the shortest processes were located in the circumventricular organs, and cells with the most ramified processes were found in gray matter regions. Microglial density varied as much as fivefold, with the highest numbers of microglia being found in 

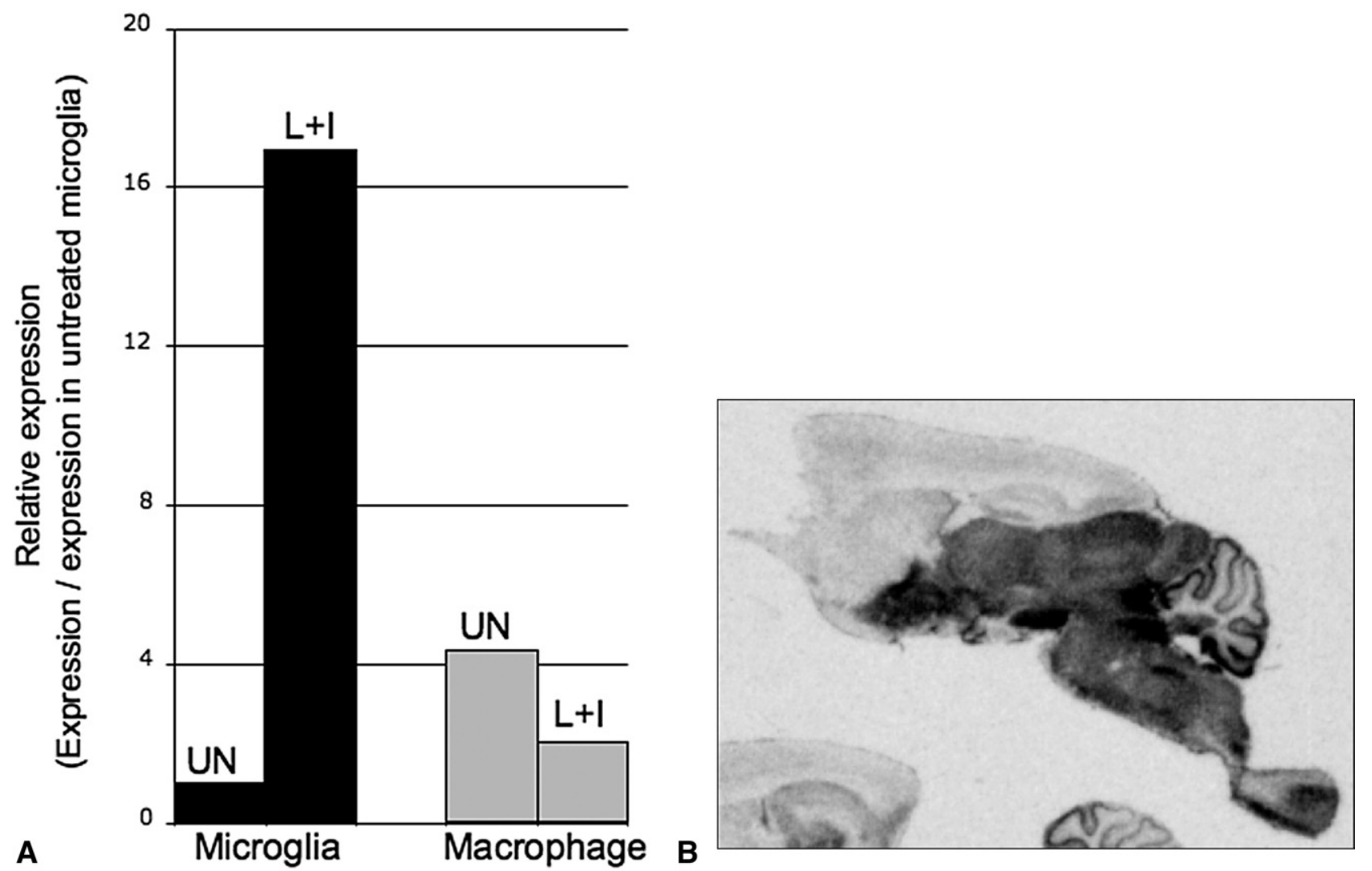

FIG. 1. Differential SPARC glycoprotein expression as a function of cell type and brain region. (a) Quantitative real-time PCR analysis was used to compare the expression of untreated (UN) and LPS-IFN $\gamma$-treated $(L+I)$ primary neonatal microglia and peritoneal macrophages. (b) SPARC expression (darker stain) in the adult rodent CNS is seen in a sagittal section from an untreated mouse. Cell preparation and technical methodologies for in situ hybridization are as previously described by Schmid et al. ${ }^{46}$

gray matter sites such as the hippocampus, olfactory telencephalon, basal ganglia, and substantia nigra. The lowest density of microglia was found in myelinated fiber tracts, including the cerebellum and the brainstem, and intermediate densities of microglia were observed in the cerebral cortex, thalamus, and hypothalamus. In contrast to microglia in nonmyelinated regions, microglia within white matter regions appear to exist in a higher basal level of activation. For example, microglia within the healthy corpus callosum express detectable levels of costimulatory molecules such as B7.2. ${ }^{51}$

Regional differences in apparent microglial phenotype extend beyond simple white matter-gray matter divisions and are now beginning to be defined in concrete molecular terms. Recently, many different research groups have profiled microglia gene expression using various cell lines, primary fetal and neonatal microglia and ex vivo adult CNS microglia and macrophages. $^{3,46,47,52,53}$ Their choice of models has strongly influenced the observed molecular fingerprint. When taken together, however, these studies are beginning to form a rudimentary molecular definition of CNS-resident microglia that can be applied to the defining microglial subpopulations in vivo.
For example, as part of our ongoing studies contrasting gene expression between CNS microglia and peripheral macrophages, we have identified several molecules that show strong regional expression. In Figure 1, comparative real-time PCR analysis illustrates that, when analyzed as a population, primary neonatal microglia express much higher levels of SPARC glycoprotein (the acronym stands for secreted protein, acidic and rich in cysteine) than do peritoneal macrophages. When examined within the adult murine CNS by in situ hybridization analysis (FIG. 1) or by Northern blot analysis (data not shown), however, SPARC expression is very low in forebrain areas of the cortex, striatum, and olfactory bulb. By contrast, SPARC expression is very high in all cells (including microglia) located in the thalamus, hypothalamus, and brainstem.

Although the functional significance of this differential expression has not been explored, previous studies have implicated increased SPARC expression with tissue remodeling during wound healing. ${ }^{54}$ These data indicate that microglia may share common regional gene expression patterns with cells of neuroectodermal origin, perhaps to support region-specific neuronal functions.

Region-specific specialization of microglial phenotype 
is confirmed from analysis of the spatial expression of molecules that are restricted to macrophages and are not widely expressed by all neuronal and macroglia within a region or are enriched in CNS-resident microglia (or molecules that meet both conditions). For example, in several models of neuroinflammation the orphan receptor TREM2 (triggering receptor expressed on myeloid cells-2) is expressed at 10-fold higher levels on purified CD45 $5^{\text {low/intermediate }}$ resident microglia than on acutely infiltrating CD45 ${ }^{\text {high }}$ macrophages. ${ }^{46,47,55}$

As analyzed by in situ hybridization analysis, however, not all microglia express detectable levels of TREM2. ${ }^{46}$ The highest percentage of TREM2-expressing microglia and the highest level of TREM2 expression per cell were observed in regions associated with susceptibility to amyloid pathology, such as the cingulate and entorhinal cortices. ${ }^{55}$ Strikingly, human knockout data suggests that TREM2-expressing microglia play an essential (but to date undefined) role in maintaining normal neuronal function. ${ }^{56-58}$ Using positional cloning, Paloneva et al. ${ }^{56}$ identified that the absence of a functional TREM2 system in humans was the cause of NasuHakola disease, a disorder characterized by early onset cognitive dementia.

Although human knockout studies illustrate the necessity of TREM2-expressing microglia in maintaining cortical neuronal function, they do not reveal which TREM2-triggered microglial functions are essential for preventing symptomatic Nasu-Hakola disease. To date, rodent studies by several groups have demonstrated only that TREM2 increases macrophage immune function. $^{56-58}$ Specifically, in peripheral macrophages and dendritic cells, TREM2-mediated activation increases both phagocytosis and the expression of molecules required to present antigen to $\mathrm{CD} 4^{+} \mathrm{T}$ cells (MHC class II, CD40, B7.2, and CCR7). ${ }^{59,60}$ Stimulus-induced increases in antigen-presenting cell activity usually correlate with increased susceptibility to generate proinflammatory $\mathrm{CD} 4{ }^{+} \mathrm{T}$ cells. Both Takahashi et al. ${ }^{61}$ and Piccio et al., ${ }^{62}$ however, demonstrated by blocking TREM 2 or overexpressing TREM2 in EAE that this pathway promotes myeloid-mediated inhibition of T-cell responses (perhaps by antigen presentation-mediated generation of inhibitory $\mathrm{T}$ cells?).

From this brief discussion, and considering only a few expression markers, a high degree of heterogeneity in the molecular fingerprint of microglia is implied. These data suggest several obvious yet for now unanswered questions:

To what extent do these molecular profiles represent regional adaptations diverging from a common microglial precursor?
Alternatively, to what extent do these molecularly distinct profiles represent microglial differentiation from several different macrophage lineages?

Lastly, how stable and how dependent are these different phenotypes on continual interactions with the CNS?

The answers to these questions are likely to have a significant effect on understanding the cause and effect relation of microglial activation in chronic CNS neurodegenerative diseases. Microglial age-related dysfunction has been proposed as a significant cause or contributing factor underlying age-related CNS neurodegenerative diseases such as Alzheimer's disease. ${ }^{63}$ To date, however, no extensive documentation of age-related changes in the molecular profile of all CNSresident macrophage populations (parenchymal and perivascular) has been done. Nonetheless, microglia from mice and humans display increasing signs of structural deterioration, display higher expression levels of a handful of classic macrophage activation markers (MHC and costimulatory molecules), and progressively develop replicative senescence as a function of age, and their inflammatory responses to stimulus become hyperresponsive. ${ }^{63}$ Ideally, it would be informative to replace the aged microglial populations with bone marrow derived from young mice. To date, no models exist for eliminating nonproliferating resident microglia; thus, such experiments cannot yet be performed. It is surprising that irradiation chimeric studies have not been used to provide a rejuvenated young peripheral immune system by supplementing aged rodents with bone marrow from juvenile or young adult rodents. At the very least, such studies would indicate whether the perivascular macrophage phenotype is dependent primarily on the health of the cells themselves or on that of the CNS microenvironment.

\section{HOW TO MODEL AND TEST THE CONSEQUENCES OF HETEROGENEITY}

Ideally, the function of these different populations of microglia and macrophages would be tested extensively in specific in vivo and in vitro assays. Unfortunately, in vivo experiments do not lend themselves to detailed study of receptor triggered intracellular events and precise quantitation of specific effector functions. Conversely, the results from in vitro culture may not always yield data predictive of in vivo biology, presumably due to the absence in vitro of phenotype-determining CNS environmental signals provided by healthy active neurons and macroglia.

For example, it is well documented in several different in vitro assay systems that cultured microglia, when stimulated with LPS and IFN $\gamma$ in vitro develop a potent 
neurotoxic phenotype. ${ }^{25,46}$ In contrast, intracerebral injections of LPS and IFN $\gamma$ provoke robust but self-resolving recruitment of peripheral macrophages into the CNS coupled with robust activation of microglia throughout the CNS. In vivo, no neuronal death is observed at the doses injected. ${ }^{25,46}$ Furthermore, when LPS-activated microglia are isolated from the CNS and added for 2 days to 2-week-old embryonic hippocampal neuron cultures, no neurotoxicity is observed, nor is there any change to the dendrite numbers and morphology (FIG. 2 and Bilousova et al., unpublished data). Note that peripheral-derived macrophages isolated from the same injected brains did cause dramatic reduction in dendrite numbers and shape.

\section{SUMMARY}

Almost any common macrophage marker (e.g., F4/80, CD11b, or Iba-1) will label nearly all the macrophage cells located within the healthy CNS. Acute insults to the CNS lead to rapid activation of microglia, most often characterized by morphological transformations and induction of MHC. This common pattern has led many to assume that microglia are largely a single population of homogeneous cells, and that activation proceeds along a linear progression of increasing pathogen defense and neurotoxicity. Recent data, however, have shown that the CNS-resident population can be divided into two very large populations with very different recent histories: recently blood-derived or long-term CNS residents. Histological analysis coupled with molecular profiling fur-

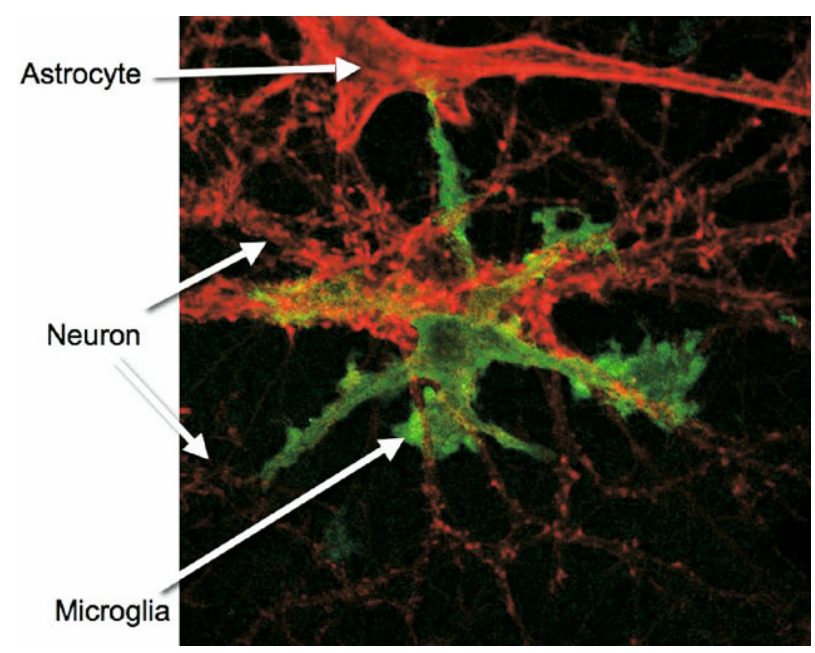

FIG. 2. Microglia activated in vivo by intracerebral injection of LPS-IFN $\gamma$ are not neurotoxic. Activated microglia isolated 24 hours after intracerebral injection of LPS-IFN $\gamma$ were isolated by flow cytometric cell sorting as previously described in Carson et al. ${ }^{44}$ and added to 2-week-old cultures of embryonic hippocampal neurons prepared as previously described in Bilousova et al. ${ }^{64}$ After 2 days of in vitro culture, microglia (labeled in green with CD11b) show no toxic effects on neurons and astrocytes (visualized in red, using phalloidin-labeled actin).

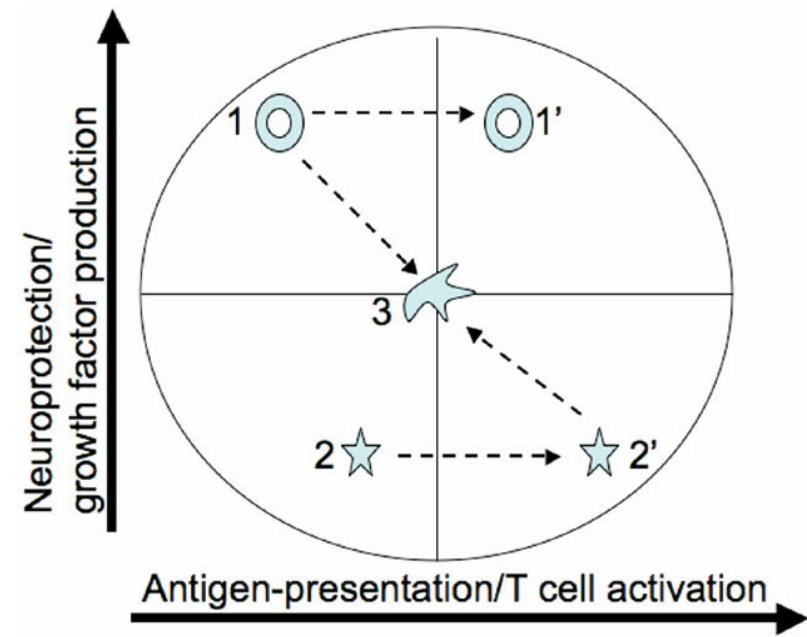

FIG. 3. Microglial activation is more than just an on-off switch or linear rheostat of responses. Microglial heterogeneity is likely an adaptation to support the diverse types of neurons present in the CNS. The heterogeneous array of basal phenotypes allows the CNS to select from a large menu of potential responses when confronted with the same activation or pathogenic stimulus. For example, hypothetical microglial cells 1 and 2 begin with very different basal levels of neuroprotective and antigen-presenting cell function. After activation with the identical stimulus, the final effector states of these two cell types differ. Similarly, two different cell types (1 and $\left.2^{\prime}\right)$ in response to different stimuli may contribute to the generation of a nearly identical effector function (cell type 3).

ther reveal broad heterogeneity even among these two populations.

This newer characterization of microglial heterogeneity suggests that activation cannot be considered linear, nor will the same stimulus cause all microglia subtypes to generate the same effector function (FIG. 3). Rather, microglial effector function is probably tailored to both brain region and stimulus. These data do complicate the development of simple therapies designed to target and inhibit all microglia. Nonetheless, such data may help in developing therapies that specifically target destructive aberrant microglial phenotypes without altering the beneficial functions of adaptive microglial phenotypes.

Acknowledgments: M.J.C. is supported by grants from the National Institute of Neurological Disorders and Stroke (NS045735, NS039508), BiogenIdec, and an unrestricted gift from Merck. I.M.E. is supported by grants from the National Institute of Mental Health (MH067121), the University of California and the FRAXA Research foundation. B.M. is a postdoctoral fellow of the National Multiple Sclerosis Society.

\section{REFERENCES}

1. Rezaie P, Male D. Mesoglia \& microglia: a historical review of the concept of mononuclear phagocytes within the central nervous system. J Hist Neurosci 2002;11:325-374.

2. Köbbert C, Apps R, Bechmann I, Lanciego JL, Mey J, Thanos S. Current concepts in neuroanatomical tracing. Prog Neurobiol 2000;62:327-351.

3. Carson MJ, Thrash JC, Lo D. Analysis of microglial gene expres- 
sion: identifying targets for CNS neurodegenerative and autoimmune disease. Am J Pharmacogenomics 2004;4:321-330.

4. Bechmann I. Failed central nervous system regeneration: a downside of immune privilege? Neuromol Med 2005;7:217-228.

5. Betmouni S, Perry VH, Gordon JL. Evidence for an early inflammatory response in the central nervous system of mice with scrapie. Neuroscience 1996;74:1-5.

6. Stoll G, Jander S. The role of microglia and macrophages in the pathophysiology of the CNS. Prog Neurobiol 1999;58:233-247.

7. Bauer J, Huitinga I, Zhao W, Lassmann H, Hickey WF, Dijkstra $\mathrm{CD}$. The role of macrophages, perivascular cells, and microglial cells in the pathogenesis of experimental autoimmune encephalomyelitis. Glia 1995;15:437-446.

8. Prineas JW, Kwon EE, Cho ES, et al. Immunopathology of secondary-progressive multiple sclerosis. Ann Neurol 2001;50:646657.

9. Streit WJ. Microglial response to brain injury: a brief synopsis. Toxicol Pathol 2000;28:28-30.

10. Shaw JA, Perry VH, Mellanby J. MHC class II expression by microglia in tetanus toxin-induced experimental epilepsy in the rat. Neuropathol Appl Neurobiol 1994;20:392-398.

11. Bjartmar C, Kinkel RP, Kidd G, Rudick RA, Trapp BD. Axonal loss in normal-appearing white matter in a patient with acute MS. Neurology 2001;57:1248-1252.

12. Trapp BD, Bo L, Mork S, Chang A. Pathogenesis of tissue injury in MS lesions. J Neuroimmunol 1999;98:49-56.

13. Peterson JW, Bo L, Mork S, Chang A, Trapp BD. Transected neurites, apoptotic neurons, and reduced inflammation in cortical multiple sclerosis lesions. Ann Neurol 2001;50:389-400.

14. Banati RB, Newcombe J, Gunn RN, et al. The peripheral benzodiazepine binding site in the brain in multiple sclerosis: quantitative in vivo imaging of microglia as a measure of disease activity. Brain 2000;123 ( Pt 11):2321-2337.

15. Allen IV, McQuaid S, Mirakhur M, Nevin G. Pathological abnormalities in the normal-appearing white matter in multiple sclerosis. Neurol Sci 2001;22:141-144.

16. Butovsky O, Landa G, Kunis G, et al. Induction and blockage of oligodendrogenesis by differently activated microglia in an animal model of multiple sclerosis. J Clin Invest 2006;116:905-915.

17. Cardona AE, Pioro EP, Sasse ME, et al. Control of microglial neurotoxicity by the fractalkine receptor. Nat Neurosci 2006;9: 917-924.

18. Nimmerjahn A, Kirchhoff F, Helmchen F. Resting microglial cells are highly dynamic surveillants of brain parenchyma in vivo. Science 2005;308:1314-1318.

19. Neumann H, Misgeld T, Matsumuro K, Wekerle H. Neurotrophins inhibit major histocompatibility class II inducibility of microglia: involvement of the p75 neurotrophin receptor. Proc Natl Acad Sci U S A 1998;95:5779-5784.

20. Mott RT, Ait-Ghezala G, Town T, et al. Neuronal expression of CD22: Novel mechanism for inhibiting microglial proinflammatory cytokine production. Glia 2004;46:369-379.

21. Hoek RM, Ruuls SR, Murphy CA, et al. Down-regulation of the macrophage lineage through interaction with OX2 (CD200). Science 2000;290:1768-1771.

22. Neumann H. Control of glial immune function by neurons. Glia 2001;36:191-199.

23. Raivich G. Like cops on the beat: the active role of resting microglia. Trends Neurosci 2005;28:571-573.

24. Davalos D, Grutzendler J, Yang G, et al. ATP mediates rapid microglial response to local brain injury in vivo. Nat Neurosci 2005;8:752-758.

25. Melchior B, Puntambekar SS, Carson MJ. Microglia and the control of autoreactive T cell responses. Neurochem Int 2006;49:145153.

26. Shepard JL, Zon LI. Developmental derivation of embryonic and adult macrophages. Curr Opin Hematol 2000;7:3-8.

27. Levison SW, Druckman SK, Young GM, Basu A. Neural stem cells in the subventricular zone are a source of astrocytes and oligodendrocytes, but not microglia. Dev Neurosci 2003;25:184196.

28. Vallières L, Sawchenko PE. Bone marrow-derived cells that pop- ulate the adult mouse brain preserve their hematopoietic identity. J Neurosci 2003;23:5197-5207.

29. Hirasawa T, Ohsawa K, Imai Y, et al. Visualization of microglia in living tissues using Iba1-EGFP transgenic mice. J Neurosci Res 2005;81:357-362.

30. Kaur C, Hao AJ, Wu CH, Ling EA. Origin of microglia. Microse Res Tech 2001;54:2-9.

31. Tsuchiya T, Park KC, Toyonaga S, et al. Characterization of microglia induced from mouse embryonic stem cells and their migration into the brain parenchyma. J Neuroimmunol 2005;160: $210-218$.

32. Kennedy DW, Abkowitz JL. Kinetics of central nervous system microglial and macrophage engraftment: analysis using a transgenic bone marrow transplantation model. Blood 1997;90:986993.

33. Lassmann H, Schmied M, Vass K, Hickey WF. Bone marrow derived elements and resident microglia in brain inflammation. Glia 1993;7:19-24.

34. Matsumoto Y, Fujiwara M. Absence of donor-type major histocompatibility complex class I antigen-bearing microglia in the rat central nervous system of radiation bone marrow chimeras. J Neuroimmunol 1987;17:71-82.

35. Becher B, Durell BG, Noelle RJ. IL-23 produced by CNS-resident cells controls $\mathrm{T}$ cell encephalitogenicity during the effector phase of experimental autoimmune encephalomyelitis. J Clin Invest 2003;112:1186-1191.

36. Hickey WF, Kimura H. Perivascular microglial cells of the CNS are bone marrow-derived and present antigen in vivo. Science 1988;239:290-292.

37. Wehner T, Bontert M, Eyupoglu I, et al. Bone marrow-derived cells expressing green fluorescent protein under the control of the glial fibrillary acidic protein promoter do not differentiate into astrocytes in vitro and in vivo. J Neurosci 2003;23:5004-5011.

38. Bechmann I, Kwidzinski E, Kovac AD, et al. Turnover of rat brain perivascular cells. Exp Neurol 2001;168:242-249.

39. Stalder AK, Ermini F, Bondolfi L, et al. Invasion of hematopoietic cells into the brain of amyloid precursor protein transgenic mice. J Neurosci 2005;25:11125-11132.

40. Simard AR, Soulet D, Gowing G, Julien JP, Rivest S. Bone marrow-derived microglia play a critical role in restricting senile plaque formation in Alzheimer's disease. Neuron 2006;49:489_ 502.

41. Serpe CJ, Kohm AP, Huppenbauer CB, Sanders VM, Jones KJ. Exacerbation of facial motoneuron loss after facial nerve transection in severe combined immunodeficient (scid) mice. J Neurosci 1999;19:RC7.

42. Byram SC, Carson MJ, Deboy CA, Serpe CJ, Sanders VM, Jones $\mathrm{KJ} . \mathrm{CD} 4^{+} \mathrm{T}$ cell-mediated neuroprotection requires dual compartment antigen presentation. J Neurosci 2004;24:4333-4339.

43. Sedgwick JD, Schwender S, Imrich H, Dorries R, Butcher GW, ter Meulen V. Isolation and direct characterization of resident microglial cells from the normal and inflamed central nervous system. Proc Natl Acad Sci U S A 1991;88:7438-7442.

44. Carson MJ, Reilly CR, Sutcliffe JG, Lo D. Mature microglia resemble immature antigen-presenting cells. Glia 1998;22:72-85.

45. Priller J, Flugel A, Wehner T, et al. Targeting gene-modified hematopoietic cells to the central nervous system: use of green fluorescent protein uncovers microglial engraftment. Nat Med 2001;7:1356-1361.

46. Schmid CD, Sautkulis LN, Danielson PE, et al. Heterogeneous expression of the triggering receptor expressed on myeloid cells-2 on adult murine microglia. J Neurochem 2002;83:1309-1320.

47. Bédard A, Tremblay P, Chernomoretz A, Vallières L. Identification of genes preferentially expressed by microglia and upregulated during cuprizone-induced inflammation. Glia 2007;55:777-789.

48. Haynes SE, Hollopeter G, Yang G, et al. The P2Y12 receptor regulates microglial activation by extracellular nucleotides. Nat Neurosci 2006;9:1512-1519.

49. Lawson LJ, Perry VH, Dri P, Gordon S. Heterogeneity in the distribution and morphology of microglia in the normal adult mouse brain. Neuroscience 1990;39:151-170.

50. Lawson LJ, Matyszak MK, Perry VH. Lessons from microglia in special sites. Clin Neuropathol 1993;12:310-313. 
51. Carson MJ. Microglia as liaisons between the immune and central nervous systems: functional implications for multiple sclerosis. Glia 2002;40:218-231.

52. Moran LB, Duke DC, Graeber MB. The microglial gene regulatory network activated by interferon-gamma. J Neuroimmunol 2007; 183:1-6.

53. Lund $\mathrm{S}$, Christensen $\mathrm{KV}$, Hedtjärn $\mathrm{M}$, et al. The dynamics of the LPS triggered inflammatory response of murine microglia under different culture and in vivo conditions. J Neuroimmunol 2006; 180:71-87.

54. Reed MJ, Puolakkainen P, Lane TF, Dickerson D, Bornstein P, Sage EH. Differential expression of SPARC and thrombospondin 1 in wound repair: immunolocalization and in situ hybridization. J Histochem Cytochem 1993;41:1467-1477.

55. Carson MJ, Doose JM, Melchior B, Schmid CD, Ploix CC. CNS immune privilege: hiding in plain sight. Immunol Rev 2006;213: $48-65$.

56. Paloneva J, Autti T, Raininko R, et al. CNS manifestations of Nasu-Hakola disease: a frontal dementia with bone cysts. Neurology 2001;56:1552-1558.

57. Daws MR, Sullam PM, Niemi EC, Chen TT, Tchao NK, Seaman WE. Pattern recognition by TREM-2: binding of anionic ligands. J Immunol 2003;171:594-599.

58. Kiialainen A, Hovanes K, Paloneva J, Kopra O, Peltonen L. Dap12 and Trem2, molecules involved in innate immunity and neurodegeneration, are co-expressed in the CNS. Neurobiol Dis 2005; 18:314-322.

59. Bouchon A, Hernández-Munain C, Cella M, Colonna M. A DAP12-mediated pathway regulates expression of CC chemokine receptor 7 and maturation of human dendritic cells. J Exp Med 2001;194:1111-1122.

60. Cella M, Buonsanti C, Strader C, Kondo T, Salmaggi A, Colonna M. Impaired differentiation of osteoclasts in TREM-2-deficient individuals. J Exp Med 2003;198:645-651.

61. Takahashi K, Prinz M, Stagi M, Chechneva O, Neumann H. TREM2-transduced myeloid precursors mediate nervous tissue debris clearance and facilitate recovery in an animal model of multiple sclerosis. PLoS Med 2007;4:e124.

62. Piccio L, Buonsanti C, Mariani M, et al. Blockade of TREM-2 exacerbates experimental autoimmune encephalomyelitis. Eur J Immunol 2007;37:1290-1301.

63. Streit WJ. Microglia and neuroprotection: implications for Alzheimer's disease. Brain Res Brain Res Rev 2005;48:234-239.

64. Bilousova TV, Rusakov DA, Ethell DW, Ethell IM. Matrix metalloproteinase-7 disrupts dendritic spines in hippocampal neurons through NMDA receptor activation. J Neurochem 2006;97:44-56. 